\title{
The Ox-Foot-Model for Investigating Endoluminal Thermal Treatment Modalities of Varicosis Vein Diseases
}

\author{
Ronald Sroka ${ }^{1}$, Kathrin Weick ${ }^{1}$, Stefanie Steckmaier ${ }^{2}$, Bernd Steckmaier ${ }^{2}$, Radka Blagova ${ }^{1,2}$, \\ Ina Sroka ${ }^{1}$, Mojtaba Sadeghi-Azandaryani ${ }^{2}$, Johann Maierl ${ }^{3}$, and Claus-Georg Schmedt ${ }^{2,4}$ \\ ${ }^{1}$ Laser-Forschungslabor, LIFE-Centre, LMU-Munich, Munich, Germany; ${ }^{2}$ Dept. of Surgery and Vascular Surgery, \\ LMU-Munich, Munich, Germany; ${ }^{3}$ Dept. of Animal Anatomy, University Bern, Bern, Switzerland; ${ }^{4}$ Klinik für Gefäßchirurgie, \\ Diakonie-Klinikum Schwäbisch Hall, Schwäbisch Hall, Germany
}

\begin{abstract}
Summary
The introduction of technical surgical innovations in clinical medicine is preceded by preclinical evaluation of prototypes. Surgical aspects such as energy dependent tissue response and tissue sealing to reduce bleeding are usually investigated in animal experiments. Extra-corporal organ models can provide the required experimental information without harming animals and thus reduce or even replace in vivo experiments. Here we describe the ex vivo ox-foot-model, which can be used for surgical investigations and for training purposes.

In the ox-foot-model the vein remains in its anatomical bed under reproducible experimental conditions, i.e., blood perfusion, blood pressure, and temperature. Innovative endoluminal surgical procedures using laser light and radio frequency for varicosis treatment were tested. Treatment parameters were investigated systematically in a large number of samples. A standardized preclinical testing procedure could be established and optimized on the basis of acute macroscopic and histological findings.

Further, optical coherence tomography could be evaluated as a time-saving diagnostic tool.

The ox-foot-model is suitable for training surgical techniques relevant for the treatment of varicosis veins. It is a cost-effective alternative to conventional in vivo experiments, providing standardized experimental conditions and reproducible experimental results while respecting the Principles of Humane Experimental Techniques: Reduction, Refinement, and Replacement of animal experiments.
\end{abstract}

Keywords: ox-foot-model, 3Rs, surgical education, varicosis vein, endoluminal treatment

\section{Introduction}

Despite great efforts and progress in the treatment of insufficient varicosis veins, nearly every second adult is afflicted with varicosis, and about $10-15 \%$ show clinical effects. The variety of side effects of conventional treatment methods, such as surgical stripping and its duration of recovery, brings a high socioeconomic burden, including non-productive time and the longterm psychological burden on the suffering patient, resulting in a demand for new and refined treatment modalities to reduce these problems. Thus, innovative and alternative treatment concepts must be considered, developed, tested, and evaluated up to clinical approval.

The protection and welfare of animals used for scientific purposes in the development of innovative medical treatment is challenging, especially considering that approval of use and CE-certification of new medical technical products, e.g., by the
FDA, requires testing of the devices in clinically adapted situations. Therefore, in addition to technical construction and realization of prototype devices for endoluminal energy application, preclinical investigations, mainly in animal experiments, are prerequisites before entering into the human clinical situation. The European Directive on the protection of animals used for scientific purposes $(E C, 2010)$ seeks to stimulate the development of alternatives to animal experiments in the sense of the 3Rs Principle: Replace, Reduce, and Refine the use of animals. Organ models have the potential to reduce animal experiments in the investigation of innovative pharmaceutical treatment modalities and in surgical interventions examining acute tissue effects. They do not require the same lengthy approval process as animal experiments, do not pose ethical concerns, and are cheaper to perform.

The ox-foot-model (OFM) was introduced and established at the Department of Vascular Surgery and the Laser-Forschungs-

Received May 8, 2012; accepted in revised form September 17, 2012 
labor at the Hospital of the University of Munich (Schmedt et al. 2006, 2007). It was developed for the assessment of techniques of endoluminal energy application, e.g., radio frequency and laser light, both suitable for thermal destruction of the vessel structures, offering an innovative procedure to treat patients suffering from varicosis veins and the resulting acute tissue responses.

\section{The ox-foot-model}

\section{Anatomy of the ox foot}

The subcutaneous cranial veins of the ox foot, $V$. saphena lateralis and $V$. digitalis dorsalis communis III and $I V$, being $20-25 \mathrm{~cm}$ in length and $5-8 \mathrm{~mm}$ in diameter, are comparable to human leg veins and allow for introduction of the endoluminal energy application systems used in the clinical situation. Similar to the anatomy in humans, the nerves $N$. fibularis superficialis and $N$. digitalis dorsalis com. III and IV lie in the immediate neighborhood of the epifascial veins. Human leg veins and ox foot veins are also similar in their histological structure. The vein wall consists of three layers, i.e., the Tunica intima, T. media and T. adventitia. The T. intima consists of the Lamina endothelialis, covered by endothelial cells, and the Stratum subendotheliale, which shows fluffily connective and elastic fibers. In place of the powerful membrane Membrana elastica interna in arteries, the veins show a net of elastic fibers (Rete elastica). The adjacent Tunica media consists of a net of helical oriented smooth muscle fibers, collagen bundles, and elastic fibers parallel to the vessel's lumen. This architecture gives the vein its elasticity and contractility. The Tunica adventitia consists mainly of connective tissue, and it passes over into the surrounding supportive tissue. As in human veins, vein valves are placed at regular distances (Blagova et al., 2007).

\section{Ox feet and blood}

Ox hind feet and blood were obtained as byproducts of slaughter in an abattoir. As the animals were killed for food purposes an application for exceptional permission for the use of animal by-products for scientific purposes according to article 17 (1) Regulation (EC) No 1069/2009 at Landeshauptstadt München, Abfallwirtschaftsbetrieb München, Fachbereich Tierkörperbeseitigung, Abteilung Verwaltung und Recht, was submitted and accepted. The special conditions of the Commission Regulation (EU) No. 142/2011, Annex VI, Chapter I, Section 1 for samples for scientific and other purposes were strictly observed. Authorized members of the academic staff of the Faculty of Medicine of the University of Munich were officially approved to request and receive animal organs, solely for the purpose of experimental and surgical investigations. As the left hind foot may be mechanically damaged by restraint, only the unhurt right hind foot of freshly slaughtered oxen (age 18-24 months, weight $550-650 \mathrm{~kg}$ ) was used for purposes of laser and radio frequency assisted vein surgery. Preparation was started within 2 hours of the animal's death, and experiments were performed within 6 hours of harvesting. Blood was collected upon slaughter in
$50 \mathrm{ml}$ syringes prepared with 5000 UE Heparin-Na (Ratiopharm GmbH, Ulm, Germany) in $5 \mathrm{ml}$ saline solution (BraunMelsungen, Melsungen, Germany) to prevent clotting (Schmedt et al., 2006, 2007; Sroka et al., 2006; Beck et al., 2007).

\section{Preparation of the ox foot}

The ox foot was prepared according to the clinical situation modeled in the experiment. Here a situation for direct vision of the vein under investigation is described.

First the skin is incised on the cranial side of the foot parallel to the hoof (Fig. 1A). A second incision is performed medially alongside the leg axis (Fig. 1B). This must be done very carefully, as the tissue below the skin must be left unharmed. Thereafter the skin is detached from the underlying tissue from medial to lateral (Fig.1C). Small skin vessels are cut immediately under the skin to prevent bleeding (Fig. 1D). Then the epifascial veins are prepared carefully. The V.digitalis dorsalis propr III is prepared and lifted carefully (Fig. 1E). A cannula (18G, Introscan Safety, Braun-Melsungen AG, Melsungen, Germany) is introduced into the vein lumen through a small incision at the distal (hoofed) end of the vein. The lumen is rinsed with saline until the saline emerging proximally is clear (Fig. $1 \mathrm{~F})$. The free passage of the vein is tested by pushing a flexible Terumo-guide wire (Cook-Medical GmbH, Mönchengladbach, Germany) in a sheath through the vein lumen (Fig. 1G). A second cannula (18G, Introscan Safety, Braun-Melsungen AG, Melsungen, Germany) for rinsing during the experiment is introduced into the $V$. digitalis dorsalis com. $I V$ (Fig. $1 \mathrm{H}$ ) and is fixed by a suture. Then the cannula in the main vein is exchanged for a sheath (Avanti-Introducer, Cordis Corporation, Miami, USA) for introduction of endolumial treatment devices. The 3-way-plug valve of this sheath allows introduction of different catheters as well as coupling to an infusion system for rinsing with saline or blood. The infusion system (Ivautrans40, Biotest Medizintechnik AG, Großostheim, Germany) is connected to a pressure sleeve to regulate the pressure. During experiments the vein pressure was stabilized to $8 \mathrm{~mm} \mathrm{Hg}$.

Figure 2 shows the completely prepared, ready-for-experiment ox-foot-model. The 3-way plug valve is first connected to a $50 \mathrm{ml}$ syringe filled with rinsing solution, dependent on the experimental conditions, which is passed through the vein; thereafter the syringe can be exchanged for the infusion system. Under these conditions different physiological parameters (here: vein pressure, tissue at room temperature, blood, and rinsing solution) can be maintained. As heat generation within the vein depends also on the cooling potential of the rinsing fluid, blood, saline, and tissue were kept at the same starting temperature (room temperature $18-20^{\circ} \mathrm{C}$ ). Furthermore, as laser light was used as energy source, the optical properties of the rinsing fluids also are of interest. Thus, using saline as a clear medium was compared to the clinical situation in which blood with its absorbing properties is present.

After surgical preparation a variety of different treatment modalities can be tested with endoluminal energy application. The energy application device (radio frequency: Closure Plus or Closure Fast, Covidien-Deutschland $\mathrm{GmbH}$, Neustadt a.d. 


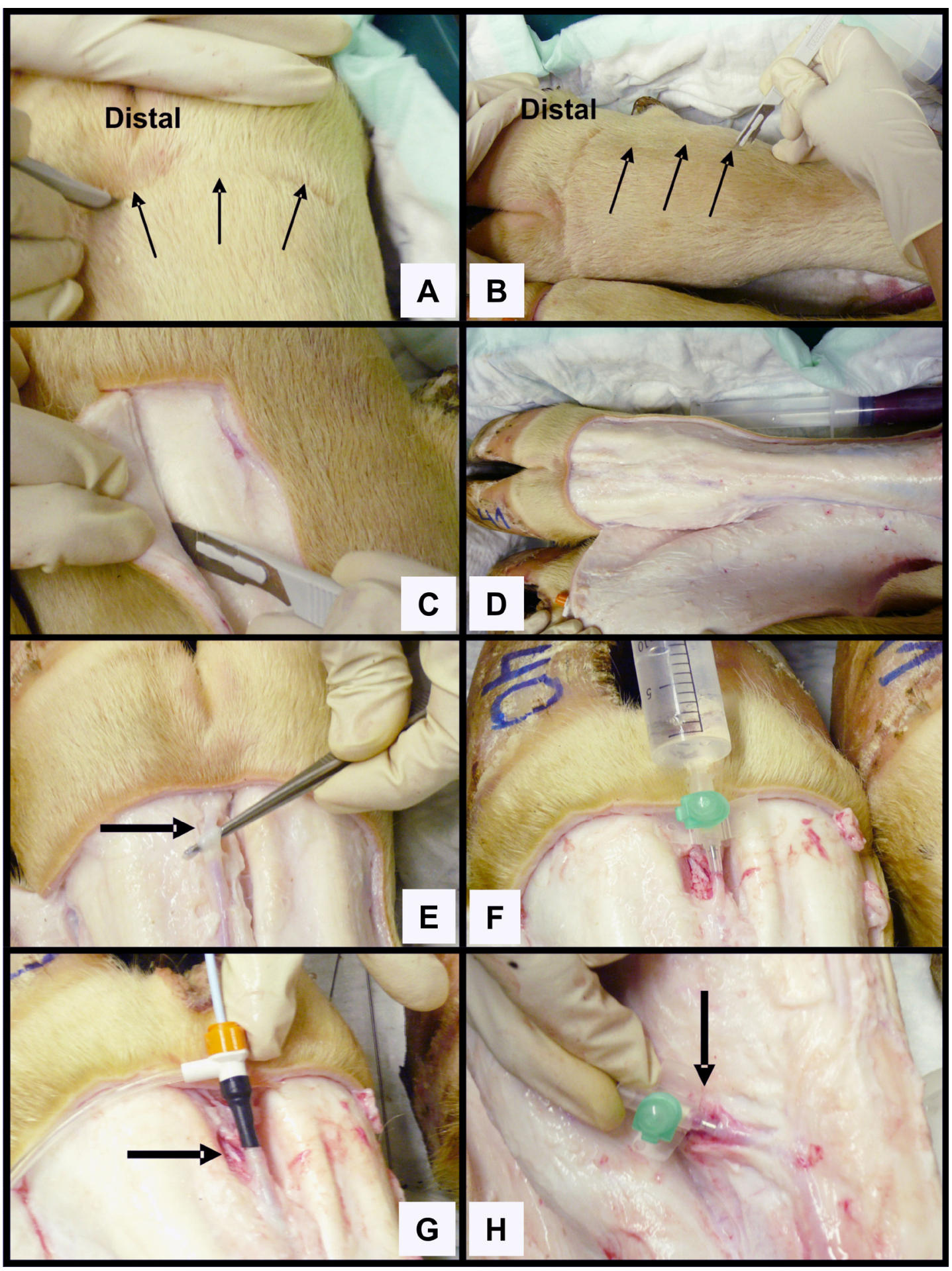

Fig. 1: Surgical preparation of the ox-foot for the experiments

(A) Incision at distal and medial side of the ox-foot parallel to hoof and (B) alongside leg axis. (C) Uncovering the skin of the ox-foot to (D) expose the underlying structures such as the vein. $(E)$ Preparation of the $V$. digitalis dorsalis propr III and smooth lifting of the vein for $(F)$ introduction of the cannula for rinsing. (G) Introduction of a sheath to guide a Terumo-guide wire and $(\mathrm{H})$ additional rinsing through the V. digitalis dorsalis com. IV.

Donau, Germany; laser, e.g.: ELVeS-Radial, biolitec GmbH, Jena, Germany) is introduced into the sheath entrance from the peripheral vein end (hoof) and pushed toward the central vein end (joint). In case of no direct vision to the vein (without skin removal) the introduction process is guided under ultrasound control, as in the clinical situation, using a conventional clinical ultrasound device. The energy application is performed as in the typical clinical situation by pulling the energy application device at a defined speed, thus travelling through the vein from the central to the peripheral section of the vein. The success of the treatment is controlled by macroscopic assessment, microscopic evaluation of hematoxylin/eosin $(\mathrm{H} / \mathrm{E})$ stained samples, or endoluminal optical coherence tomography (OCT) (M2x, LightLab, Essex, UK). OCT is an optical interferometer technique 


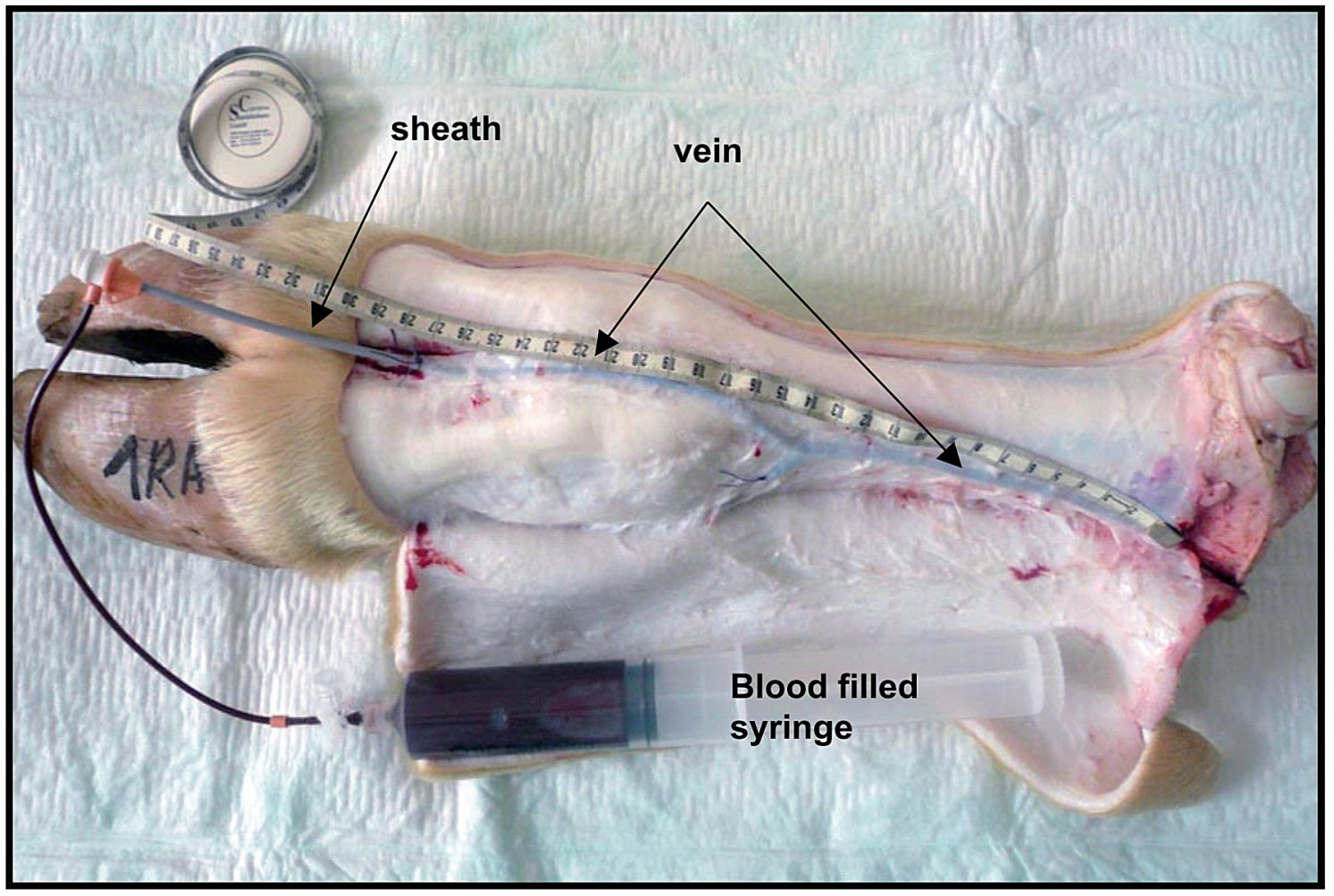

Fig. 2: OFM including sheath and blood-filled syringe ready for experiment

based upon the detection of light, backscattered from tissue layers, thus providing images of tissue layers with a resolution of 10-20 $\mu \mathrm{m}$. Due to the optical properties of tissue, the limit of the depth is about $1.5 \mathrm{~mm}$. Thus this technique shows potential for superficial diagnostics with high microscopic resolution instead of ultrasound with much larger tissue penetration but a limited resolution of about $100 \mu \mathrm{m}$.

\section{Use of the OFM for technical developments}

Optimization of endoluminal laser light treatment of veins In a first experimental setup the evaluation and optimization of clinical endoluminal laser treatment situations using clinical radiofrequency and laser-bare fiber techniques and protocols (Sroka et al., 2006; Beck et al., 2007) are described. Endoluminal laser assisted treatment of varicosis veins in a clinical situation was started using the bare fiber technique, applying $8 \mathrm{~W}$ laser energy spot-wise at $3 \mathrm{~mm}$ intervals (Navarro et al., 2001). This procedure results in perforation of the vein wall, as shown in Figure 3A, which correlates with clinical side effects reported, such as hematomas (Elmore and Lackey., 2008; Luebke and Brunkwall, 2008). In comparison, using the endoluminal RFtechnology (Closure Plus, VNUS-Medical Technology GmbH, Weinstadt, Germany) on the OFM, a whitish colored coagulated vein can be observed without signs of perforation or charring, as shown in Figure 3B (Beck et al., 2007; Blagova et al., 2007). This should be the aim of endoluminal energy deposition for thermal destruction of varicosis veins.

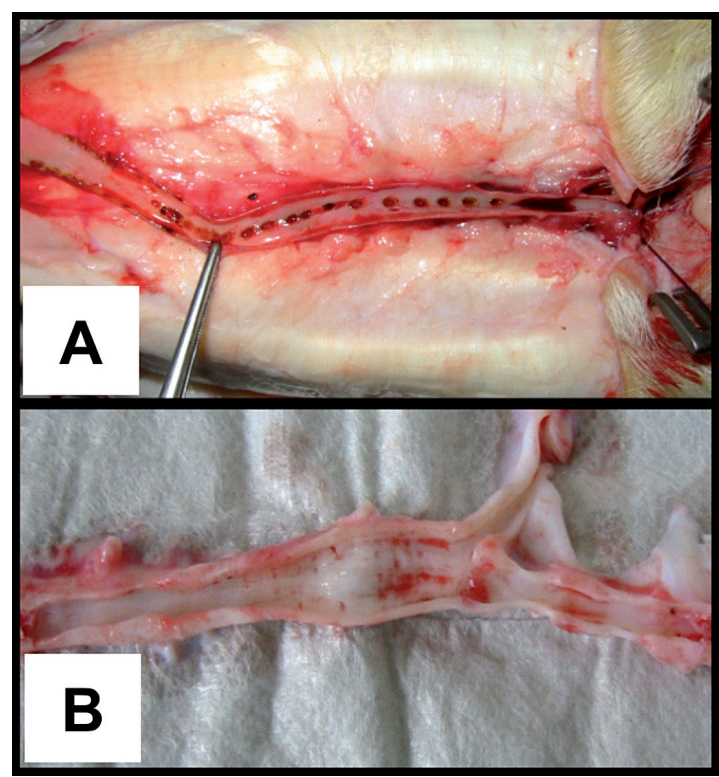

Fig. 3: Surgically opened vein in OFM after endoluminal treatment

(A) Endoluminal laser treatment using the bare fiber single spot $3 \mathrm{~mm}$ depart technique in OFM. (B) Endoluminal Radiofrequency Closure Plus - technique, harvest from OFM for further macroscopic and histologic examination. 

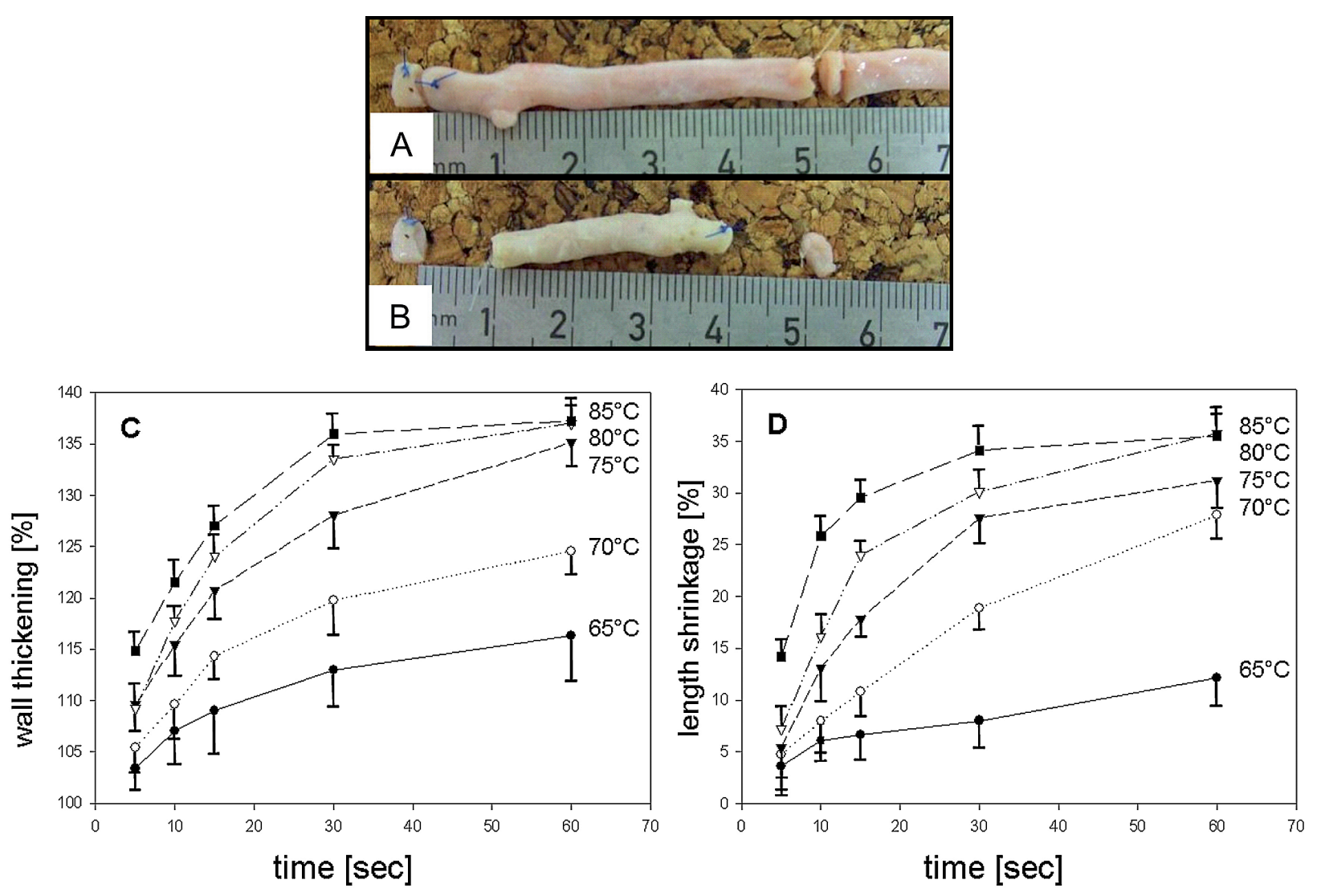

Fig. 4: Exposure to thermal heat induces shrinkage of the vein tissue

(A) $5 \mathrm{~cm}$-sample immediately before and (B) after thermal exposure $\left(\mathrm{T}=85^{\circ} \mathrm{C}, 30 \mathrm{sec}\right)$. The thickening of the vein wall (C) and the shrinkage (D) are shown for temperatures between 65 and $85^{\circ} \mathrm{C}$ and exposure durations of 5 to 60 sec. Each point reflects the mean of 5 individual experiments and SD.

Based upon these systematic investigations on the macroscopic tissue effects of laser light energy application using the bare fiber technique and their histological correlates, further approaches could be developed and investigated on the OFM. In a first step, the bare fiber technique was changed to fibers with radial laser light emission, either in a ring or cylindrical shape. Thus the light was emitted directly to the vein wall and, in addition, the irradiance could be reduced below the ablation threshold (Sroka et al., 2010). In a second step, the wavelength could be optimized, thus targeting the tissue water instead of the hemoglobin (Sroka et al., 2010) because of the different optical absorption spectra of these liquids. The aim of the treatment is the direct heating of the tissue, which can be achieved by targeting the tissue water as absorber, instead of by indirect heating of the tissue by first heating the surrounding blood and thus secondarily heating the vein tissue due to heat condution. After testing several further parameters in the OFM, an improved clinical protocol could be evaluated and introduced into clinical studies (Pannier et al., 2009; Sroka et al., 2010).

Based upon the knowledge of the principal parameters of endoluminal laser light application, in this case wavelength
$1470 \mathrm{~nm}$, laser power $6-8 \mathrm{~W}$, pull velocity $1 \mathrm{~mm} / \mathrm{s}$, the parameters for clinical use could be defined. Thus, in the current clinical protocol for laser assisted endoluminal varicosis treatment using the radial emitting fiber technique, only the laser power was slightly increased to $10-15 \mathrm{~W}$, leaving the other parameters constant. Clinical studies showed relevant success with reduced side effects (Pannier et al., 2010, 2011; Maurins et al., 2009).

\section{Optimization of thermal energy treatment of veins}

In a further approach, the investigation of thermal-induced effects on vein tissue by endoluminal laser treatment (Beck et al., 2007) could be performed. The effects of thermal energy application on the vein tissue could be investigated using explanted OFM-veins. Shrinkage of vein tissue after thermal exposure ( 65 to $85^{\circ} \mathrm{C}$ for various time durations) and the increase in vein wall thickness are shown in Figure 4A and B. Reproducibility and minor influence of physiological changes of the OFMtissue due to time duration between harvesting the ox-feet and performing the experiment could be obtained from the results of these experiments. Figure $4 \mathrm{C}$ and D show the response of the vein to heat induction $\left(65\right.$ to $\left.85^{\circ} \mathrm{C}\right)$ for different time spans 


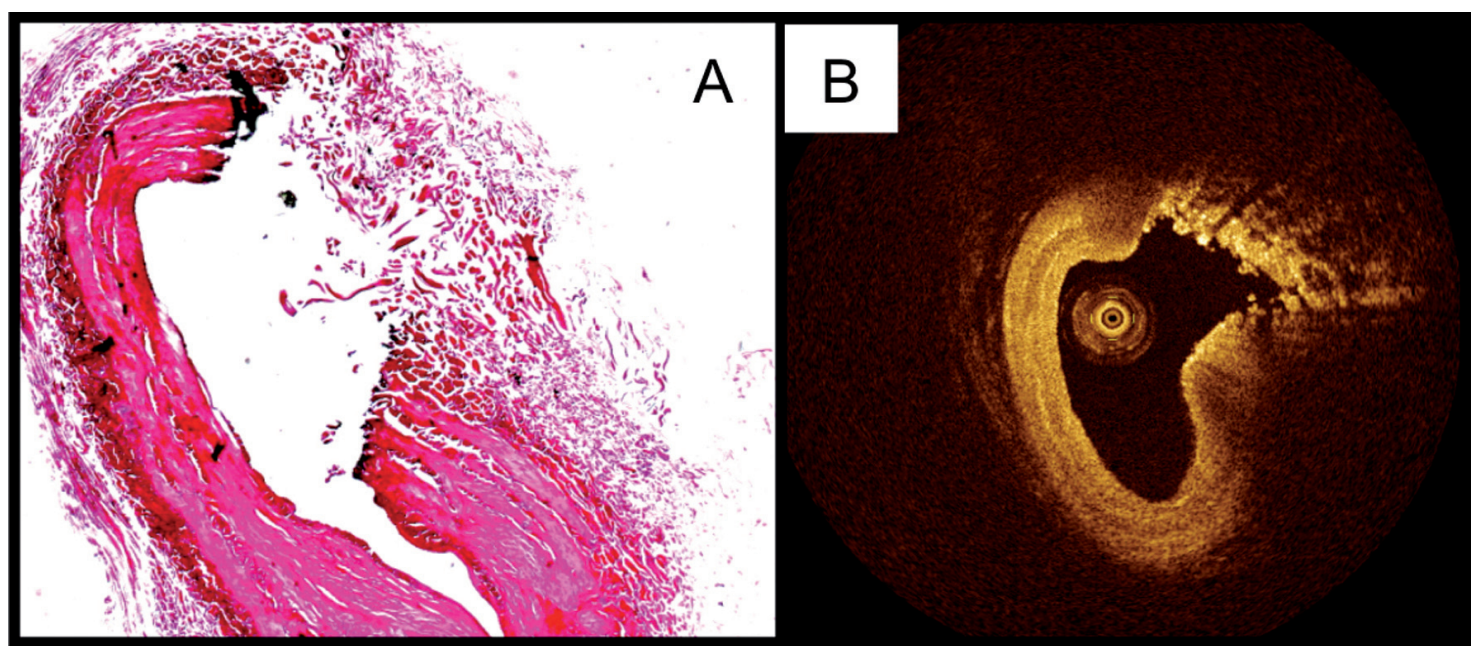

Fig. 5: Comparison of microscopic vein wall layer

(A) Laser-affected structure using classical tissue histology of HE-staining after harvesting the tissue from the body. (B) Innovative endoluminal in-situ generated images by means of OCT-techniques show the same structure with a resolution of $20 \mu \mathrm{m}$.

( 5 to $60 \mathrm{sec}$ ). The result of this experiment showed that after heat induction at $85^{\circ} \mathrm{C}$ for $30 \mathrm{sec}$, a maximum of wall thickening and shrinkage could be obtained.

\section{Further technical developments}

Additional investigations using the OFM as a reproducible setup results in optimization of the laser approach by development of innovative energy application systems (Blagova et al., 2007; Sroka et al., 2010). Based on the experimental experiences, the OFM was successfully used as a varicosis treatment model to investigate innovative endoluminal treatment procedures in a comparable manner. Radiofrequency obliteration (Proebstle et al., 2008; Luebke and Brunkwall, 2008), endoluminal laser treatment (Proebstle et al., 2002; Pannier et al., 2009; Ravi et al., 2006), and endoluminal steam procedures (van den Bos et al., 2010) were tested, and different energy application devices, as well as parameters, were optimized.

In a further set of experiments using the OFM, the principle of the basic function of the endoluminal treatment procedures could be identified. It could be verified that the different forms of energy and energy application techniques result in different principles of energy absorption, heat development, and, finally, tissue response development (Sroka et al., manuscript under preparation).

\section{Use of the OFM to test usefulness of optical coherence tomography}

Finally, new and innovative optical technologies, such as optical coherence tomography (OCT), could be tested as a tool for in situ on-line structure layer visualization, thus reducing ex vivo histology investigations (Sroka et al., 2006). Histological examination of tissue is a time consuming and expensive aspect of such developments. In the OFM, the comparison of HE-stained tissue slides after tissue explantation to in situ images generated by means of endoluminal optical coherence tomography (OCT) techniques, as shown in Figure 5, clearly demonstrates the same situation of the vein wall as a layered tissue structure, and demonstrates carbonized/coagulated tissue parts and perforations. In this way, the innovative OCT technique also could be evaluated for its potential in clinical use as an in situ diagnostic tool.

\section{Discussion and outlook}

New techniques for endoluminal energy application allow adjustment of manifold parameters, but these possible combinations need to be tested and investigated in a systematic manner. With respect to laser light application and thermal radiofrequency, theoretical models and simulation programs based on light and heat transport equations in tissue are available and can partly support first reference points for the physiological responses of such methods and parameters (Mordon et al., 2007). But these tissue responses must also be investigated experimentally, especially with regard to potential bleeding or sealing of the tissue. Therefore, investigations cannot be performed in cell-culture experiments; however investigations in defined and standardized extra-corporal organ models are a good choice. They allow the investigation of multiple parameters and parameter combinations under reproducible conditions, which is impossible in animal experiments. This way, animal experiments for this purpose (Kim et al., 2004; Zimmet and Min, 2003; Goldman and Detwiler, 2003) can be reduced and sometimes completely avoided.

We developed the ox-foot-model for the investigation of endoluminal treatment techniques of varicosis veins. Ox feet are byproducts in the abattoir and are available in large numbers upon request at low cost. By using the ox feet from the abattoir no animals needed to be killed for the purpose of this study.

The regimentation and standardization of breeding and animal husbandry make oxen and cattle a quasi-standardized population, allowing the definition of criteria to standardize the experi- 


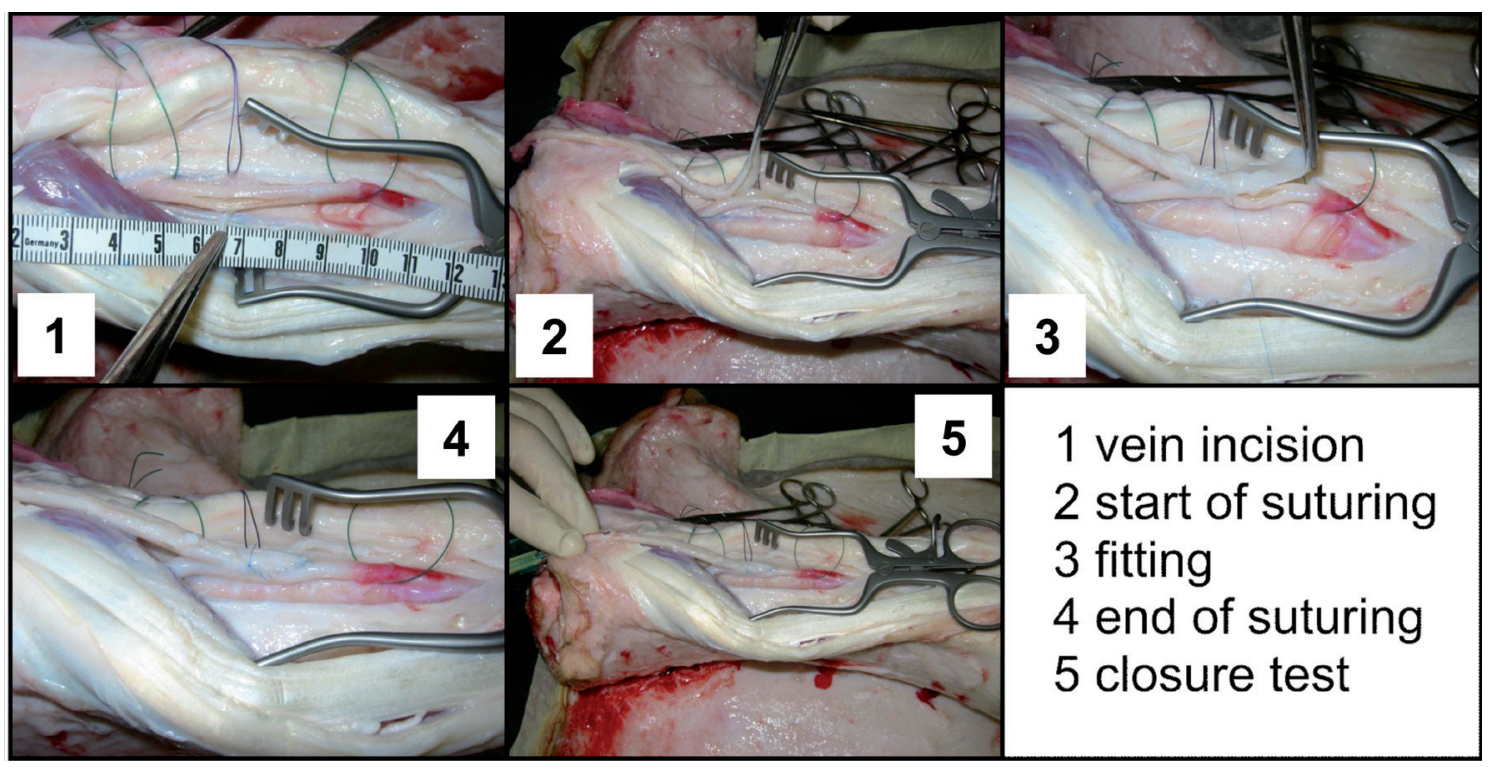

Fig. 6: Demonstration of using the OFM during surgical skill training of performing a bypass-anastomosis

(1) The surgical prepared operation area with tissue holder. The vein is open by an incision ready for adaptation of a bypass vessel. (2) Smooth fixation of the bypass vessel to the incision. (3) Complete and secure fitting of the anastomosis, and (4) finish of suturing. (5) Afterwards a closure test could be performed by pushing colored saline through the bypass-vessel through the anastomosis area, thus to confirm closure.

ments, i.e., right hind foot, specific age and weight, defined time between harvesting of organ and experiment. The preparation of the ox-foot can be realized with minor effort in a manageable time. The isolation of the organ also reduces the influence of animal-individual physiological preconditions. A good coordination with the abattoir thus allowed the investigation of a broad range of variables in multiple samples followed by statistical evaluation.

Of course, the organ-model also has limitations. The reaction of the whole organism cannot be studied; the tissue ages after the animal's death, limiting the time in which an experiment can be performed; long-term effects of the treatment cannot be observed; and despite the anatomical and functional analogy, there are differences between humans and oxen. Therefore, the results of the experiments cannot be transferred to the clinical situation without restriction.

An alternative to performing experiments on animal organmodels is the use of human tissue explants harvested during operations (Proebstle et al., 2002). Although those experiments look promising, such tissue has its own set of limitations: it is available on a much smaller scale, the explants may be affected by disease, and as the explants are detached, immediate effects on the directly adjacent tissue (i.e., perivascular tissue) cannot be taken into consideration.

Apart from the use of the OFM to test and optimize technical developments, it could be put to use in the quality management of the production of medical products in accordance with industrial standards like ISO13485 and EN9001.

The OFM is further suitable for the teaching and skill training of physicians. The OFM was introduced for training the process of endoluminal treatment, including orientation and finding the vessel by means of surgical dissection, use of ultrasound guidance, introduction of the sheath, positioning of the guide wire, introduction of the laser fiber by means of the Seldinger-technique, and introduction of the local tumescence anesthesia under ultrasound guidance. After energy application the veins could be surgically prepared to macroscopically assess the result. To demonstrate the immediate effects, the veins were prepared prior to energy application. To date, endoluminal therapeutic treatment techniques have been demonstrated in laboratory setups, as well as in workshops, e.g.: "Endovenous Treatment Modalities - Hands-On using the Ox-Foot-Model" at the $52^{\text {nd }}$ Jahrestagung der Deutschen Gesellschaft für Phlebologie in 2010. The OFM also has been used for training in surgical ligation and bypass-anastomosis techniques of vessels, and it shows potential for investigations concerning vascular tissue soldering (Bogni et al., 2009). As shown in Figure 6, the surgical education on the OFM for training the techniques of bypass-anastomosis or arterio-venous fistulas for dialysis could be easily performed under standardized conditions. Here too, the OFM can support a highly motivated training situation, either with or without blood perfusion.

Taken together, the standardized ox-foot-model could be used for cost effective optimization of technical developments, as well as for surgical skill-training purposes. Investigations such as endoluminal treatment of varicosis veins could be realized without harming animals, thus respecting the 3Rs. While the OFM offers many advantages over experiments on human tissue explants and living animals, only acute tissue responses can be observed. Further investigations and developments are under way to improve the clinical situation by on-line monitoring possibilities during endoluminal treatment. 


\section{References}

Beck, T. J., Burgmeier, C., Blagova, R., et al. (2007). Thermalinduced effects on vein tissue - a basic ex-vivo investigation for EVLT. Medical Laser Applications 22, 238-241.

Blagova, R., Burgmeier, C., Steckmeier, S., et al. (2007). Exvivo investigation on endoluminal laser therapy of varicosis - an optimization process. Medical Laser Applications 22, 242-247.

Bogni, S., Stumpp, O., Reinert, M., and Frenz, M. (2009). Thermal model for optimization of vascular tissue soldering. J Biophotonics 3, 284-295.

EC - European Commission (2010). Directive 2010/63/EU of the European Parliament and of the Council of 22. September 2010 on the protection of animals used for scientific purposes. Official J EU L276, 33-76.

Elmore, F. A. and Lackey, D. (2008). Effectiveness of endovenous laser treatment in eliminating superficial venous reflux. Phlebology 23, 21-31.

Goldman, M. P. and Detwiler, S. (2003). Endovenous 1064-nm and 1320-nm NdYAG laser treatment of the porcine greater saphenous vein. Cosmetic Dermatology 16, 25-28.

Kim, H. S., Liapi, E., Arepally, A., et al. (2004). Endovenous laser ablation of internal jugular vein and its effects on common carotid arteries in swine models. J Vasc Interv Radiol 15, S133.

Luebke, T. and Brunkwall, J. (2008). Systematic review and meta-analysis of endovenous radiofrequency obliteration, endovenous laser therapy, and foam sclerotherapy for primary varicosis. J Cardiovasc Surg 49, 213-233.

Maurins, U., Rabe, E., and Pannier, F. (2009). Does laser power influence the results of endovenous laser ablation (EVLA) of incompetent saphenous veins with the 1470-nm diode laser? A prospective randomized study comparing 15 and $25 \mathrm{~W}$. Int Angiol 28, 32-37.

Mordon, S. R., Wassmer, B., and Zemmouri, J. (2007). Mathematical modeling of 980-nm and 1320-nm endovenous laser treatment. Lasers Surg Med 39, 256-265.

Navarro, L., Min, R. J., and Bone, C. (2001). Endovenous laser: a new minimally invasive method of treatment for varicose veins - prelimnary observations using an $810 \mathrm{~nm}$ diode laser. Dermatol Surg 27, 117-122.

Pannier, F., Rabe, E., and Maurins, U. (2009). First results with a new 1470-nm diode laser for endovenous ablation of incompetent saphenous veins. Phlebology 24, 26-30.

Pannier, F., Rabe, E., and Maurins, U. (2010). 1470 nm diode laser for endovenous ablation (EVLA) of incompetent saphenous veins - a prospective randomized pilot study comparing warm and cold tumescence anaesthesia. Vasa 39, 249-255.

Pannier, F., Rabe, E., Rits, J., et al. (2011). Endovenous laser ablation of great saphenous veins using a $1470 \mathrm{~nm}$ diode laser and the radial fibre - follow-up after six months. Phlebology 26, 35-39.

Proebstle, T. M., Lehr, H. A., Kargl, A., et al. (2002). Endovenous treatment of the greater saphenous vein with a $940-\mathrm{nm}$ diode laser: Thrombotic occlusion after endoluminal thermal damage by laser-generated steam bubbles. J Vasc Surg 35, 729-736.

Proebstle, T. M., Vago, B., Alm, J., et al. (2008). Treatment of the incompetent great saphenous vein by endovenous radiofrequency powered segmental thermal ablation: first clinical experience. J Vasc Surg 47, 151-156.

Ravi, R., Rodriguez-Lopez, J. A., Trayler, E. A., et al. (2006). Endovenous ablation of incompetent saphenous veins: a large single-center experience. J Endovasc Ther 13, 244-248.

Schmedt, C.-G., Sroka, R., Steckmeier, S., et al. (2006). Investigation on radiofrequency and laser $(980 \mathrm{~nm})$ effects after endoluminal treatment of saphenous vein insufficiency in an ex-vivo model. Eur J Vasc Endovasc Surg 32, 318-325.

Schmedt, C.-G., Meissner, O. A., Hunger, K., et al. (2007). Evaluation of endovenous radiofrequency ablation and laser therapy with endoluminal optical coherence tomography in an ex vivo model. J Vasc Surg 45, 1047-1058.

Sroka, R., Schmedt, C.-G., Steckmeier, S., et al. (2006). Exvivo investigation of endoluminal vein treatment by means of radiofrequency and laser irradiation. Medical Laser Applications 21, 15-22.

Sroka, R., Weick, K., Sadeghi-Azandaryani, M., et al. (2010). Endovenous laser therapy - applications studies and latest investigations. J Biophotonics 3, 269-276.

van den Bos, R. R., Milleret, R., Neumann, M., and Nijsten, T. (2010). Proof-of-principle study of steam ablation as novel thermal therapy for saphenous varicose veins. J Vasc Surg. doi:10.1016/j.jvs.2010.06.171

Zimmet, E. and Min, R. J. (2003). Temperature changes in perivenous tissue during endovenous laser treatment in a swine model. J Vasc Interv Radiol 14, 911-915.

\section{Acknowledgements}

The authors would like to thank all students and trainees for their interest and motivation as well as all their ideas and questions asked to improve the OFM. Furthermore we would like to thank the coworkers of the abattoir Munich for support.

\section{Correspondence to}

Ronald Sroka, PhD

Laser-Forschungslabor

LIFE-Centre

LMU-München CAMPUS Großhadern

Marchioninistrasse 23

81377 München

Germany

Phone: +49 8970954879

Fax.: +49 8970954864

e-mail: ronald.sroka@med.uni-muenchen.de http://laser.klinikum.uni-muenchen.de/ 